\title{
Worldline numerics applied to custom Casimir geometry generates unanticipated intersection with Alcubierre warp metric
}

\author{
Harold White $^{1, \mathrm{a}}$, Jerry Vera ${ }^{1}$, Arum Han ${ }^{2,3,4,5}$, Alexander R. Bruccoleri ${ }^{6}$, Jonathan MacArthur ${ }^{6}$ \\ ${ }^{1}$ Limitless Space Institute, 16441 Space Center Blvd., Bldg. D-200, Houston, TX 77058, USA \\ ${ }^{2}$ Department of Electrical and Computer Engineering, Texas A\&M University, College Station, USA \\ ${ }^{3}$ Department of Biomedical Engineering, Texas A\&M University, College Station, USA \\ ${ }^{4}$ NanoBio Systems Lab. (nanobio.tamu.edu), College Station, USA \\ ${ }^{5}$ AggieFab (aggiefab.tamu.edu), College Station, USA \\ ${ }^{6}$ Izentis LLC, P.O. Box 397002, Cambridge, MA 02139, USA
}

Received: 17 May 2021 / Accepted: 25 July 2021 / Published online: 31 July 2021

(C) The Author(s) 2021

\begin{abstract}
While conducting analysis related to a DARPAfunded project to evaluate possible structure of the energy density present in a Casimir cavity as predicted by the dynamic vacuum model, a micro/nano-scale structure has been discovered that predicts negative energy density distribution that closely matches requirements for the Alcubierre metric. The simplest notional geometry being analyzed as part of the DARPA-funded work consists of a standard parallel plate Casimir cavity equipped with pillars arrayed along the cavity mid-plane with the purpose of detecting a transient electric field arising from vacuum polarization conjectured to occur along the midplane of the cavity. An analytic technique called worldline numerics was adapted to numerically assess vacuum response to the custom Casimir cavity, and these numerical analysis results were observed to be qualitatively quite similar to a two-dimensional representation of energy density requirements for the Alcubierre warp metric. Subsequently, a toy model consisting of a $1 \mu \mathrm{m}$ diameter sphere centrally located in a $4 \mu \mathrm{m}$ diameter cylinder was analyzed to show a three-dimensional Casimir energy density that correlates well with the Alcubierre warp metric requirements. This qualitative correlation would suggest that chip-scale experiments might be explored to attempt to measure tiny signatures illustrative of the presence of the conjectured phenomenon: a real, albeit humble, warp bubble.
\end{abstract}

\section{Background}

Work being conducted under a DARPA DSO $^{1}$ grant is investigating the implications of the dynamic vacuum model for

\footnotetext{
${ }^{1}$ Defense Advanced Research Projects Agency Defense Science Office.

a e-mail: sonny@limitlessspace.org (corresponding author)
}

the possibility of structure to the Casimir energy distribution manifested in a parallel plate cavity. The dynamic vacuum model predicts that the negative vacuum energy density present in the parallel plate cavity is not isotropic, rather there is a varying energy density field present in the cavity with an average value that corresponds with that predicted by the traditional equation for parallel-plate Casimir energy density. The structure predicted to be manifest in the cavity takes the form of a larger magnitude negative vacuum energy density concentrated along the cavity mid-plane that relaxes non-linearly to the unperturbed state at the cavity boundaries. Based on detailed studies of the atomic orbitals of the hydrogen atom, and deriving the acoustic wave equation from the Schrödinger equation $[1,2]$, it is speculated that the energy density structure in a Casimir cavity is coupled to a small polarization field in the vacuum fluctuations resulting in a small but non-zero electrostatic field originating along the cavity mid-plane and terminating at the grounded cavity walls. It has been further reasoned in the literature that it may be possible to construct a customized Casimir cavity equipped with small pillars placed at the mid-plane as depicted in Fig. 1 such that when the pillar channel is sampled by a high impedance oscilloscope, the scope would detect a transient non-zero voltage signal ${ }^{2}$ that would rapidly go to zero as the stored energy in the polarization field is depleted from the measurement process [4].

\footnotetext{
${ }^{2}$ A recently published paper [3] details an experimental campaign using an asymmetric Casimir cavity arrangement where one cavity has a small separation and the other cavity has a much larger effective separation. This experimental campaign observed a current flow from the larger cavity electrode to the smaller cavity electrode. Our cavity is analogous in that the two parallel plates define a large cavity and the plate-pillar system plays the role of the smaller cavity.
} 


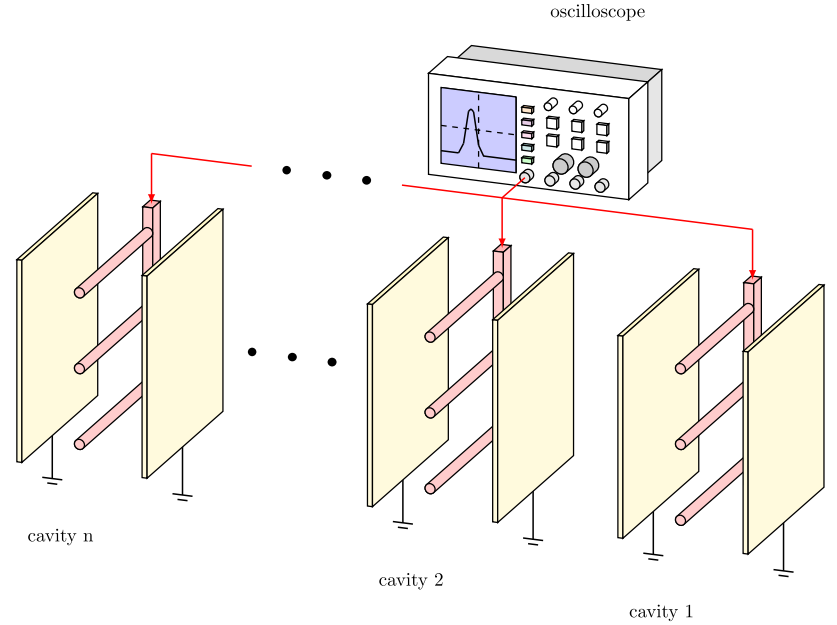

Fig. 1 Parallel plate cavity design with pillar-like features along the mid-plane - the dynamic vacuum model predicts that the scope will measure a small but non-zero transient voltage pulse from the pillars once connected to the cavities as shown. The anticipated geometry of each cavity is approximately $4 \mu \mathrm{m}$ wide with each plate measuring $40 \mu \mathrm{m}$ by $40 \mu \mathrm{m}$. The pillars are expected to be approximately $1 \mu \mathrm{m}$ in diameter

If these cavities can be made small enough and arrayed together in large enough numbers to increase the stored energy, the magnitude and duration of this predicted transient voltage signal may be detectable in a laboratory setting. The equation that predicts the magnitude of this small but non-zero electrostatic field was derived in [4] and takes the following form: $E=\sqrt{\hbar c \pi^{2} / 1080 \epsilon_{0} d^{4}}$. A quick calculation for a Casimir cavity with a $4 \mu \mathrm{m}$ gap predicts a magnitude of the electrostatic potential arising from the polarization of the vacuum fluctuations along the mid-plane of the cavity to be $\sim 0.7 \mathrm{mV}$. Figure 2 shows some examples of the current nanofabrication trian runs that are ongoing as part of the effort. The left panel in the figure depicts a recent result obtained utilizing a Nanoscribe $3 \mathrm{~d}$ printer to evaluate a range of cavity gaps and pillar sizes. The material being used in the manufacture is SU-8 2025 permanent epoxy negative photoresist. After printing and curing, the cavities then undergo electroless plating to add a layer of $\mathrm{Ag}$ to the outer surfaces. The right panel shows recent etching results using Deep Reactive Ion Etching (DRIE) equipment to etch the high-aspect ratio cavity planes into a silicon wafer substrate. This approach uses standard $\mathrm{SiO}_{2}$ wafer materials with the expectation that the final concepts will have a metallic layer added by means of evaporation. The results depicted were focused on achieving the high-aspect ratio etch, and future work will incorporate the masking necessary to produce pillars. A technical concern with the proposed design implementation is how the presence of pillars might affect the predicted Casimir energy density distribution within the cavity - would there be self-screening that occurs within the pillar

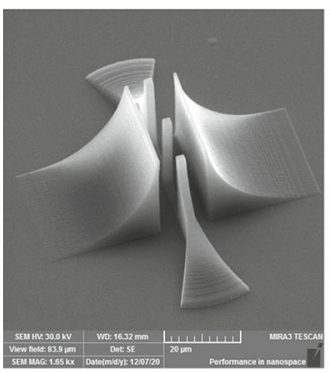

(a) Nanoscribe 3D printer cavity sample

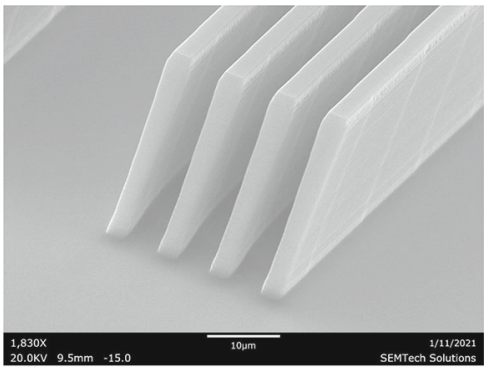

(b) DRIE high-aspect ratio etching results
Fig. 2 Initial results from current nanofabrication trian runs: the left panel in the figure depicts a recent result obtained utilizing a Nanoscribe 3D printer to evaluate a range of cavity gaps and pillar sizes; the right panel shows recent etching results using Deep Reactive Ion Etching (DRIE) equipment to etch the high-aspect ratio cavity planes into a silicon wafer substrate

that minimizes the magnitude of the negative vacuum energy density present inside the pillar, and hence the magnitude of the detectable signal? It was during the analysis process seeking to address this screening question that an unanticipated intersection with the Alcubierre metric was found.

\section{Introduction}

The literature search phase of this DARPA project discovered a numerical methods approach known as worldline numerics [5-9] that can be used to study and quantify the Casimir energy density and force. The curious aspect of this modeling approach that makes it of high interest to the DARPA project is that it predicts that there is structure (spatial variation) to the negative vacuum energy density in a Casimir cavity analogous to the predictions of the dynamic vacuum model. The primary value of considering an implementation of this model technique is that it provides a high fidelity prediction of the perturbed vacuum state inside model geometry (e.g. the evanescent fields in structure) along with predictions for the perturbed vacuum state within the cavity gaps. An additional benefit of the worldline numerics method ${ }^{3}$ for studying the Casimir effect is that it can be used to address any type of geometry with effectively no restrictions on curvature or lack of smoothness.

Due to the similarities with the dynamic vacuum model and its computational flexibility coupled with maturity, this worldline numerics technique has been implemented to consider the custom Casimir cavities and determine the predicted negative vacuum energy density distribution in the cavities and within the pillars. Figure 3 depicts the numerical analysis results from our implementation of the worldline approach

\footnotetext{
${ }^{3}$ Also referred to as the loop cloud method [10].
} 


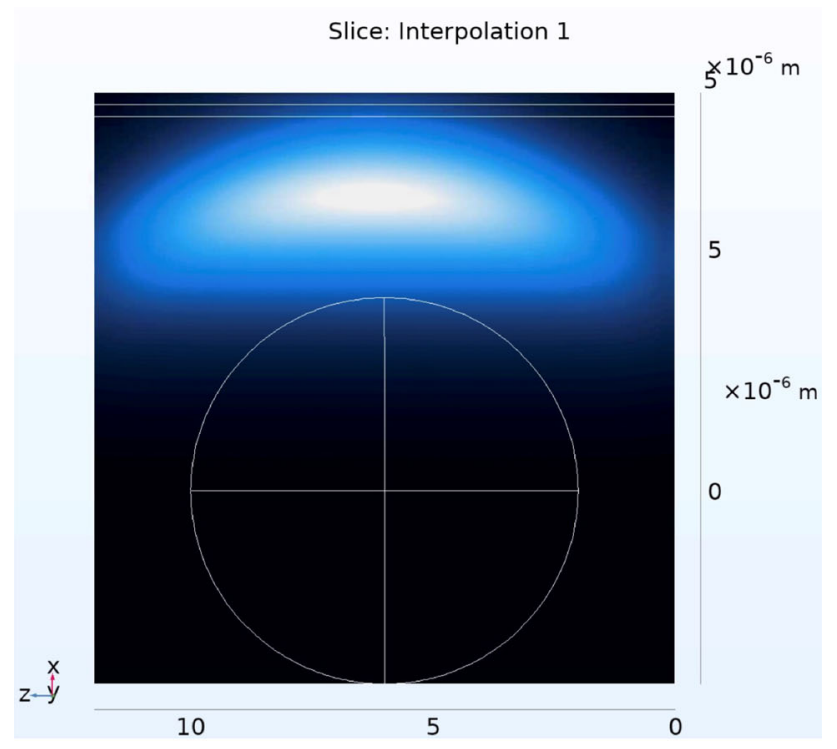

(a) 2 dimensional cut of a sphere-plate case

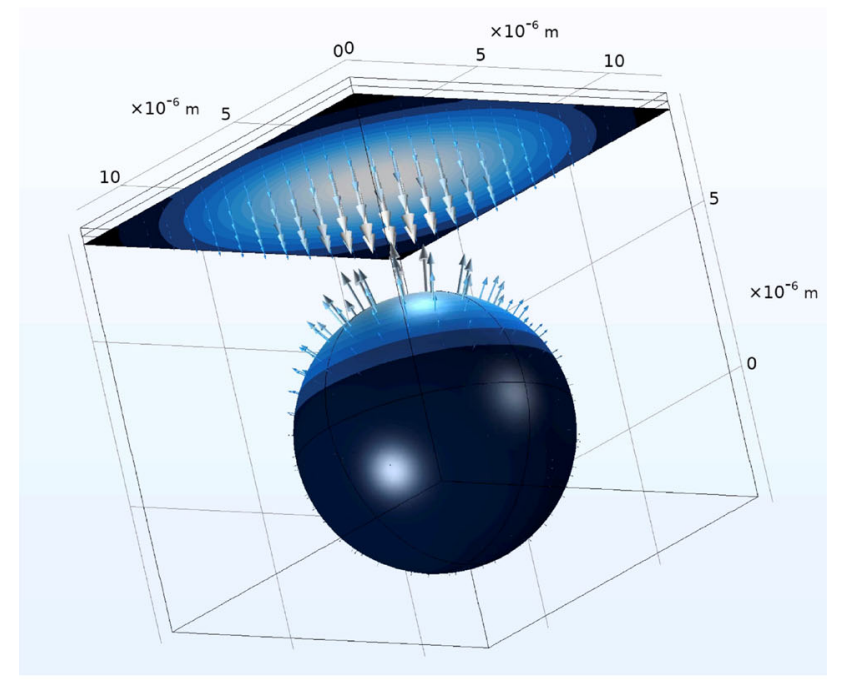

(b) importing of analysis results into COMSOL to calculate force distribution over surfaces

Fig. 3 Panel (a) shows a 2 dimensional section cut of a sphere-plate case using the numerical worldline technique. The approach was implemented using Open MPI-enabled c-code and the analysis was run on $1002.40 \mathrm{GHz}$ Intel Skylake CPUs. The model grid was a $50 \times 50 \times 50$ grid with a 2000 unit-loop ensemble. The bottom plot shows a vector plot of forces normal to model surfaces and was created by importing the worldline numerics results into COMSOL

considering a 3 dimensional sphere with a radius of $4 \mu \mathrm{m}$ separated from an infinite flat plate by a separation of $4 \mu \mathrm{m}$. The approach was implemented using Open MPI-enabled ccode and the analysis was run on $1002.40 \mathrm{GHz}$ Intel Skylake CPUs. The model grid was a $50 \times 50 \times 50$ grid with a 2000 unit-loop ensemble. The top panel shows a 2 dimensional section cut of the predicted distribution to the negative vacuum energy density between the two bodies where it should also be noted that the field gradients extend into both the body of the sphere and the flat plate in the form of evanescent fields. The bottom panel shows the distribution of forces across the surfaces of the model and was generated using COMSOL. The following section provides a brief summary of the details behind the worldline numerics analytic approach.

\section{Synopsis of Casimir worldline numerics}

The string theory inspired worldline numerics approach to determine the Casimir effect is developed in detail in [5], and the critical aspects of the analysis technique are briefly summarized here for convenience. With the objective of evaluating the Casimir interaction energy $E_{\text {Casimir }}$ (e.g. normalized) arising from the coupling of a real scalar quantum field $\phi$ of finite mass $m$ with a background potential $V(x)$ that represents the Casimir geometry, the key equation from Section 2.1 of [5] is the effective action shown in Eq. (1).

$$
\begin{aligned}
\Gamma[V]= & -\frac{1}{2(4 \pi)^{2}} \int_{1 / \Lambda^{2}}^{\infty} \frac{d T}{T^{3}} e^{-m^{2} T} \int d^{4} x \\
& \times\left[\left\langle W_{V}[y(t) ; x]\right\rangle_{y}-1\right]
\end{aligned}
$$

The expectation value in Eq. (1) is the average of the loop ensemble over all closed loops with Gaussian walks:

$$
\left\langle W_{V}[y(t) ; x]\right\rangle_{y}=\frac{\int_{y(0)=y(1)} \mathscr{D} y W_{V}[y(t) ; x] e^{-\int_{0}^{1} d t \dot{y}^{2} / 4}}{\int_{y(0)=y(1)} \mathscr{D} y e^{-\int_{0}^{1} d t \dot{y}^{2} / 4}},
$$

where the following "Wilson loop" identity has been introduced with $y$ representing the (unit) loop path ${ }^{4}, x$ representing the position shift of the unit loop in model space, and $T$ denoting the proper time and serves to scale the unit loops:

$$
W_{V}[y(t) ; x]=\exp \left[-T \int_{0}^{1} d t V(x+\sqrt{T} y(t))\right]
$$

Equipped with this information, one can calculate the (unrenormalized) Casimir energy as $\mathscr{E}=\Gamma / \int d x_{0}$ where the integral represents the "volume" in the time direction. When considering the Casimir force, the portion of the Casimir energy that has a dependency on the relative positions of the bounding geometries can be obtained by subtracting the energies of the single objects from the total Casimir energy.

$E_{\mathrm{Casimir}}:=\mathscr{E} \mathrm{V}_{1}+\mathrm{V}_{2}+\cdots-\mathscr{E} \mathrm{V}_{1}-\mathscr{E} \mathrm{V}_{2}-\cdots$

$\overline{4_{y:[0 ; 1] \mapsto}} \mathbb{R}$ and unit proper time $t \in[0,1]$. 
The Casimir force can be obtained by taking the negative spatial derivative of this interaction energy, and further, this process has removed any UV divergences. In the Dirichlet limit $\lambda \rightarrow \infty$ and for a massless scalar field with Dirichlet boundaries in $\mathrm{D}=3+1$, the worldline representation of the Casimir interaction energy boils down to ${ }^{5}$ :

$E_{\text {Casimir }}=-\frac{1}{2(4 \pi)^{2}} \int_{0}^{\infty} \frac{d T}{T^{3}} \int d^{3} x_{C M}\left\langle\Theta_{\Sigma}[\mathbf{x}(\tau)]\right\rangle_{x}$

The worldline functional $\Theta_{\Sigma}[\mathbf{x}(\tau)]=0$ if the re-scaled unit loop does not intersect any Casimir geometry and $\Theta_{\Sigma}[\mathbf{x}(\tau)]=1-n$ if the re-scaled loop intersects $n \geq 1$ Casimir geometry. The numerical evaluation process requires two discretization steps. The first is the discretization of the path integral into an ensemble of $n_{\mathrm{L}}$ random paths $\mathbf{x}_{\ell}(\tau)$, $\ell=1, \ldots, n_{\mathrm{L}}$ with each path forming a closed spacetime loop. The second is the discretization of the proper time interval $\tau \in[0, T]$ into $N$ steps such that an individual closed loop consists of $N$ points per loop: $\mathbf{x}_{\ell k}:=\mathbf{x}_{\ell}(k \cdot T / N), k=$ $1, \ldots N$. Transporting and rescaling the ensemble of unit loops to a point $\mathbf{x}_{\mathrm{CM}}$ in the model takes the following form: $\mathbf{x}_{\ell k}=\mathbf{x}_{\mathrm{CM}}+\sqrt{T} \mathbf{y}_{\ell k}$. Applying these two discretizations to the Casimir interaction energy in Eq. (5) yields the following form:

$E_{\text {Casimir }}=-\frac{1}{2(4 \pi)^{2}} \int_{0}^{\infty} \frac{d T}{T^{3}} \int d^{3} x_{\mathrm{CM}} \frac{1}{n_{\mathrm{L}}} \sum_{\ell=1}^{n_{\mathrm{L}}} \Theta_{\Sigma}\left[\mathbf{x}_{\mathrm{CM}}+\sqrt{T} \mathbf{y}_{\ell}\right]$.

As the worldline numeric approach for the Casimir phenomenon is based on (massless) scalar fields, the technique can currently only assess idealized behaviour for bounding geometry and cannot assess any frequency dependence of materials. Additionally, the approach developed to date in the literature does not account for the impacts of temperature. However, it is still a very capable and appealing technique in that it can provide quick and fairly accurate assessments for very complicated geometries where analytic techniques are not practical.

\subsection{Generating unit loops, computational approach, and implementation validation}

The developers of the worldline numerics for the Casimir phenomenon explored numerous ways [5] to generate ensembles of unit loops with Gaussian distribution ranging from a heat bath kernel to random walks, and finally landing on a technique denoted as the "v-loop" algorithm. The curious

\footnotetext{
5 A thorough articulation of the renormalization process is detailed in Section 3.2 of [8].
}

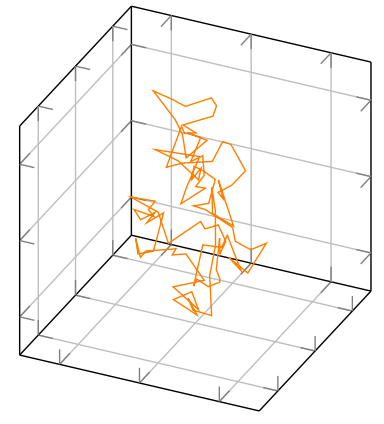

(a) 100 point unit loop

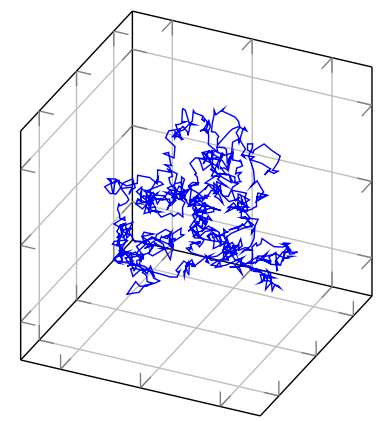

(c) 1000 point unit loop

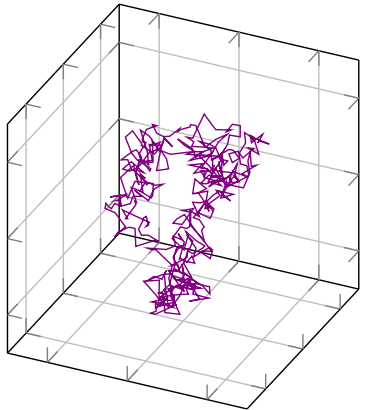

(b) 500 point unit loop

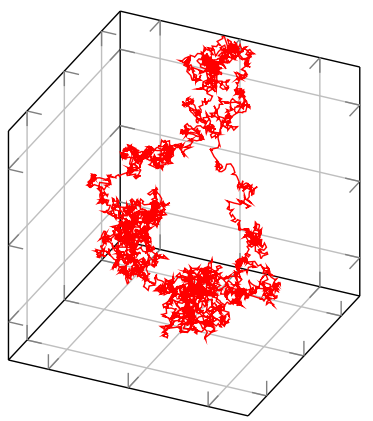

(d) 5000 point unit loop
Fig. 4 Gaussian distributed closed unit loops representative of scalar field fluctuation generated using v-loop methodology. Algorithm will generate ensemble of $n_{\mathrm{L}}$ unit loops to be scaled and applied at each geometric point of interest in a physical model

reader is encouraged to review the referenced manuscript for a thorough discussion of the benefits and shortfalls of the different techniques explored. The "v-loop" technique was selected as it can computationally generate an ensemble of $n_{\mathrm{L}}$ each having $\mathrm{N}$ points per loop without having to perform multiple iterations on each loop to realize a closed random walk/worldline with the required statistical characteristics. Figure 4 shows several examples of unit-loops generated by the v-loop methodology ranging from a 100 point unit loop to a 5000 point unit loop. A summary of the computational procedure steps are provided here to facilitate the reader's understanding of the "v-loop" approach:

1. generate $N-1$ numbers $w_{i}(i=1, \ldots, N-1)$ with a Gaussian distribution $e^{-w_{i}^{2}}$ (e.g. using Box-Müller method);

2. calculate $N-1$ numbers $\bar{v}_{i}$ by normalizing $w_{i}$ :

$$
\begin{aligned}
& \bar{v}_{1}=\sqrt{\frac{2}{N}} w_{1}, \\
& \bar{v}_{i}=\frac{2}{\sqrt{N}} \sqrt{\frac{N+1-i}{N+2-i}} \quad w_{i}, i=2, \ldots, N-1 ;
\end{aligned}
$$


3. calculate $v_{i}$ for $i=2, \ldots, N-1$ with the following:

$$
\begin{array}{r}
v_{i}=\bar{v}_{i}-\frac{1}{N+2-i} v_{i-1,1}, \\
\text { where } v_{i-1,1}=\sum_{j=2}^{i-1} v_{j} ;
\end{array}
$$

4. a unit loop $\mathbf{y}$ can now be created by using:

$$
\begin{aligned}
y_{1} & =\frac{1}{N}\left(\bar{v}_{1}-\sum_{i=2}^{N-1}\left(N-i+\frac{1}{2}\right) v_{i}\right), \\
y_{i} & =y_{i-1}+v_{i}, \quad i=2, \ldots, N-1, \\
y_{N} & =-\sum_{i=1}^{N-1} y_{i}
\end{aligned}
$$

5. this procedure is repeated $n_{\mathrm{L}}$ times to create the unit loop ensemble $\mathbf{y}_{\ell}$ with $\ell=1, \ldots, n_{\mathrm{L}}$.

The benefit of this numeric worldline approach is that it can be used to address any type of geometry while other approaches such as Proximity-Force Approximation (PFA) are not as flexible. Additionally, the approach has no dependency on the choice of model grid spacing or grid choice. The answer for a single point of interest in space does not have any interdependency on any other model grid points and may be calculated in total isolation if that is all that is needed. Figure $5^{6}$ provides a pictoral representation of the analysis process for a parallel plate Casimir cavity. As indicated in the figure, once the loop ensemble has been generated the computational process to calculate the Casimir interaction energy follows the below enumerated steps:

1. The loop ensemble is moved to each model grid point of interest and scaled using proper time until $2+$ bodies in the model are pierced;

2. the scale at which an individual loop pierces $2+$ bodies defines the integral limits for the Casimir interaction energy integral;

3. the energy at the geometric point of interest in the model is increased based on wavelength (loop scale) and loop weight factor;

4. this scaling process is repeated for each loop in the ensemble at a geometric point of interest in the model;

5. the above steps are repeated for each geometric point of interest in the model.

\footnotetext{
$\overline{6}$ While the figure depicts a regularized model grid for communication purposes, the computational result at an individual model point is not dependent on adjacent points making the technique independent of grid choice.
}

Validation of our implementation of the numeric worldline approach was done on a plate-plate case and a corresponding plate-sphere case and was compared to documented results in the literature [5]. For the reader's awareness, the work documented in [5] conducts extensive analysis to compare analytic results to the numeric results produced by the worldline technique for the simple plate-plate scenario and plate-sphere scenario. The referenced study explored the impact of number of points per unit loop, number of unit loops in an ensemble, separation distance of geometries, coupling, and mass. It is not the intention of this paper to duplicate the viability of the overall worldline numerics approach as this has already been done in the literature as noted, rather the intention of this paper is to apply this very powerful and flexible technique to fairly complicated geometries where only numerical methods can effectively be used. In our validation effort, we confirmed that our model predict the correct Casimir force for a given plate-plate or sphere place scenario, and subsequently compared their Casimir interaction energy density results from their numeric worldline algorithm to our interaction energy density results from our numeric worldline algorithm. The subsequent more complicated geometries we consider forthwith as part of this work do not have trivial analytic solutions which is why the numeric worldline technique is employed.

A plot of the results from our implementation is provided in Fig. 6 for the two cases and the plot also includes a plateblade case. The geometry of all three cases is such that the closest point of separation between all three cases is identical allowing for comparison of the results to evaluate the effects of curvature. The plots reflect the energy density as measured along a line normal to the plate-plate geometry and these geometric points of interest are the same for the plate-sphere and plate-blade cases. The colors of the Casimir energy density plots correspond to the colors of the toy geometry also overlayed on the plot facilitating comparison of the results and to clearly see the impact of curvature. Comparing our results to those in literature indicates that our algorithms are functioning properly.

\section{Analysis results and unanticipated findings}

As discussed in the opening of the manuscript, the critical concern for this project is if the presence of a pillar in the Casimir cavity would serve to screen itself in such a way that it would be unable to see the negative vacuum energy density gradient predicted to be present in the cavity if the pillars were not present. A model was built to assess a $4 \mu \mathrm{m}$ cavity with a $1 \mu \mathrm{m}$ diameter pillar placed in the middle of the cavity. The model discretization was a $35 \times 35$ grid running $\pm 4 \mu \mathrm{m}$ in both the $x$ and $y$ axis. The $x$-axis is the vector normal to the parallel cavity plates, and the $y$-axis is orthogonal to the $x$-axis and defines the 2D surface for the energy density plot. 
Fig. 5 Panel (a) shows the unit loop ensemble being moved to a geometric point of interest in model. Panel (b) shows scaling of one of the unit loops from the ensemble to the point that it intersects $2+$ bodies of model. This establishes the support $\mathscr{S}_{\ell}$ to be used in integration and determine the weighted contribution of the loop to the Casimir energy density of the vacuum at this point in the model

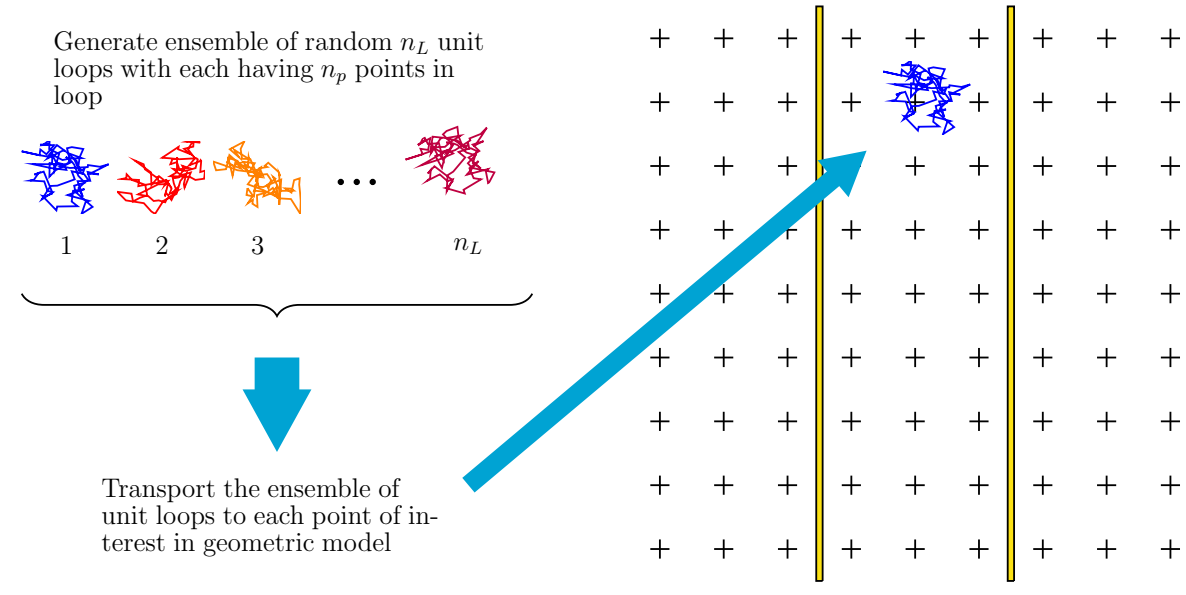

(a) transporting unit loop ensemble to geometric point of interest
For each loop in the ensemble, transport to a grid point, scale loop to determine proper time for which loop pierces $2+$ bodies

This defines the support, $\mathcal{S}_{\ell}$, of the loop that sets the integral limits for integration

The energy density at the point is then increased by a loop-weighted amount

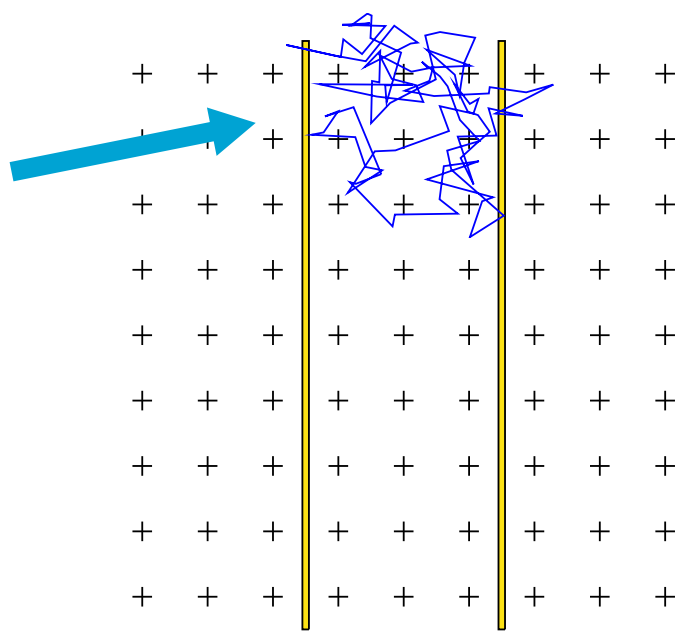

(b) scaling of an individual loop of the ensemble so that it intersects $2+$ bodies of the model
The origin of the coordinate system is at the center of the pillar. The analysis results are shown in Fig. 7 with a two dimensional representation of the energy density depicted in the left panel and a log of the energy density levels in the right panel. Inspecting the log-plot on the right shows that while the presence of the pillar in the cavity does perturb the field, it actually serves to slightly increase the effective negative vacuum energy density seen in the pillar by a factor of 35 compared to the density level present without the pillar in the cavity. These analysis results would suggest that the pillar does not adversely self-screen itself in a manner that prevents it from seeing the field magnitude in the cavity with no pillar present. Rather, due to the slightly elevated state, it could be reasoned that the pillar seems to focus the gradient in a manner that would at most allow the pillar to drain the stored energy in the cavity at a quicker rate once it is connected to a high impedance oscilloscope.This effect might result in a need for more cavities to provide enough stored energy such that the duration of the transient voltage signal will last long enough for detection.

While the analysis results discussed above are encouraging for the project objective of attempting to measure the presence of structure in the negative vacuum energy density within a customized Casimir cavity, the implications of this particular predicted negative vacuum energy density distribution is quite intriguing for an altogether different reason. As it so happens, the structure of the field around the pillar in the two dimensional plot is qualitatively very similar to a plot of the negative vacuum energy density necessary for the Alcubierre warp metric. ${ }^{7}$ Figure 9 shows a zoomed view on the numeric worldline analysis of the plate-pillar case on

\footnotetext{
7 The Casimir phenomenon was first discussed as an alternative source to exotic matter for the idea of a wormhole in $[11,12]$ and later expanded on in a book[13] on wormholes by Visser. It was also identified by Alcubierre[14] in his seminal paper as an alternative source to exotic matter for the manifestation of a warp bubble. The Casimir phenomenon
} 


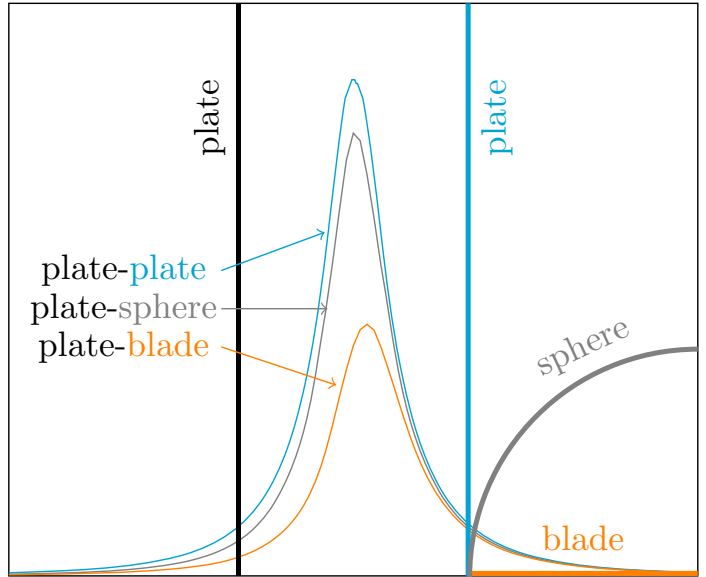

Fig. 6 Plot of validation runs for plate-plate and plate-sphere cases. The plot also includes a plate-blade case. The geometry elements are represented by a thick vertical black line for the left Casimir cavity plate, a thick vertical cyan line for the right plate, a thick gray quarter circle to show the location of the sphere, and an orange horizontal line representing the blade. The colors of the plots of energy density distribution match the color of the corresponding geometry overlayed in the plot. The magnitude of the energy density distribution clearly shows a decrease in magnitude as the cases go from plate-plate to plate-blade. Additionally, the plots show that there is a shifting of the peaks to the right due to the curvature effects

the top, and the energy density field for the Alcubierre metric on the bottom. Before fully exploring the implications of this unanticipated intersection between these two models, the critical elements of the Alcubierre model will be identified and discussed that lead to the energy density that allows the "trick" to work. The motivation for the Alcubierre metric [14] was to develop a model within the context of general relativity that would mathematically encapsulate the idea of a space warp that would allow for hyper fast travel between arbitrarily distant stellar objects. The metric and shaping function are provided in Eq. (10) (with $G=c=1$ where $v_{s}$ is the speed of the craft, $f\left(r_{s}\right)$ is the shaping function, $\sigma$ is the shell thickness parameter that controls the thickness of the warp bubble wall, and $R$ is the radius of the warp bubble.

$$
\begin{gathered}
d s^{2}=-d t^{2}+\left(d x-v_{s} f\left(r_{s}\right) d t\right)^{2}+d y^{2}+d z^{2} \\
f\left(r_{s}\right)=\frac{\tanh \left(\sigma\left(r_{s}+R\right)\right)-\tanh \left(\sigma\left(r_{s}-R\right)\right)}{2 \tanh (\sigma R)}
\end{gathered}
$$

The critical element of the model that enables stellar hyper fast transit is conjectured to be the York Time which is a measure of the expansion and contraction of space associated with the metric. ${ }^{8}$ A plot of the York Time is provided in

\section{Footnote 7 continued}

has more recently been explored by Garattini in $[15,16]$ as a sourcing material for "benign" wormholes.

${ }^{8}$ It was shown in [18] by putting the metric into canonical form that the catalytic mechanism was not the York Time, rather it was the boost field

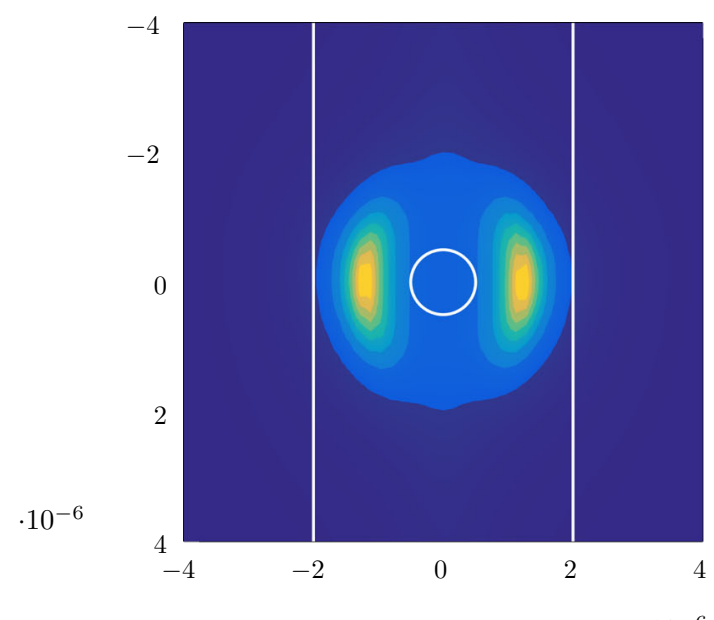

(a) linear plot of energy density

$\cdot 10^{-6}$

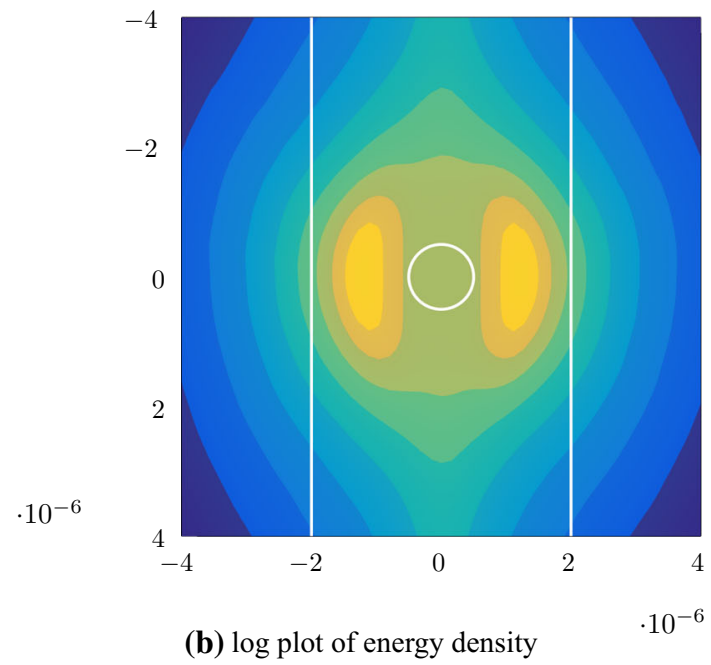

Fig. 7 The white lines represent a $4 \mu \mathrm{m}$ Casimir cavity, and the circle represents the $1 \mu \mathrm{m}$ pillar located at the midplane of the cavity. Panel (a) shows the linear 2D plot of the energy density and panel (b) shows the $\log 2 \mathrm{D}$ plot. The bright yellow regions show the impact of the presence of the pillar on the energy density, and the log plot shows that the pillars do not self-screen themselves from the background field gradient resulting from the presence of just the plates (lime-green color)

Fig. 8 [17]. The York Time field is depicted as a grid that has a wave-like appearance with a simple representation of a notional craft overlayed on top of the field to show the connection between the spacetime disturbance and the source of the negative vacuum energy density. The York Time plot indicates that space is expanding behind the spacecraft and contracting in front of the spacecraft. The craft depicted in

\section{Footnote 8 continued}

serving as a multiplier of the ship's initial velocity, akin to watching a movie in fast-forward. With the canonical form of the metric, the expansion and contraction of space is viewed as a response of spacetime as the hyperfast craft transits through space - space piles up in front of the craft and stretches out behind the craft. 
the plot has a central part located in the center of the warp bubble in the region where the spacetime is flat, the proper acceleration $\alpha$ is zero, and local clocks are synchronized with external clocks on earth. The craft is equipped with a ring structure that represents an encapsulation of exotic matter or negative vacuum energy density distributed throughout.

The York Time, $\theta$, and the energy density distribution $T^{00}$, are shown in Eqs. (11) and (12) respectively. The $v_{s}$ term again represents the velocity of the craft, $x_{s}$ represents the position of the center of the craft (and hence the fields), and the $y^{2}$ and $z^{2}$ just define a radial distance $\rho$ from the central $x$-axis. Anecdotally, a sensitivity analysis of the field equations conducted in $[19,20]$ showed that by varying the shell thickness parameter, $\sigma$, one could reduce the magnitude of the York Time, and as a result the total energy required to make the concept work. The analysis effort created two animations available online that show the response of the York Time field [21] and the energy density field [22] to variation of the shell thickness parameter. The animations show that as the warp bubble wall thickness increases the peak energy density decreases significantly, and as the warp bubble wall thickness decreases the peak energy density increases. The reasoning behind this response is that the York Time can be viewed as a sort of 3-dimensional strain of space, and as the shell thickness increases, the amount of 3-dimensional strain needed to manifest a target speed $v_{s}$ decreases which is accompanied by a decrease of energy density, and thus a reduction in total energy. This is not without a cost - as can be seen in the online animation of the York Time field, the region of flat spacetime available for the critical portions of the craft, say for a crew or science instruments, is decreased as the bubble wall thickness is increased. So the energy optimization process has a competing constraint in the form of the required size, $R$, for the warp bubble to adequately encapsulate sensitive cargo within the flat spacetime region inside the bubble wall.

$$
\begin{aligned}
\theta & =\quad v_{s} \frac{x_{s}}{r_{s}} \frac{d f\left(r_{s}\right)}{d r_{s}} \\
T^{00} & =-\frac{1}{8 \pi} \frac{v_{s}^{2}\left(y^{2}+z^{2}\right)}{4 r_{s}^{2}}\left(\frac{d f\left(r_{s}\right)}{d r_{s}}\right)^{2}
\end{aligned}
$$

Now that the Alcubierre metric has been introduced and the critical elements have been identified and discussed, a comparison between the exotic matter requirements of the warp concept and the numeric worldline analysis results for the custom Casimir cavity may now be made. The top panel of Fig. 9 shows a close up view of the predicted response of the quantum vacuum within the custom Casimir cavity, and the bottom panel shows a 2-dimensional representation of the energy density necessary for the Alcubierre model. The concentrations in the negative vacuum energy density due to the presence of the pillar in the Casimir cavity are qualitatively very similar to the $2 \mathrm{D}$ representation of the $T^{00}$ for the Alcubierre model. It should be noted that the 2-dimensional plot

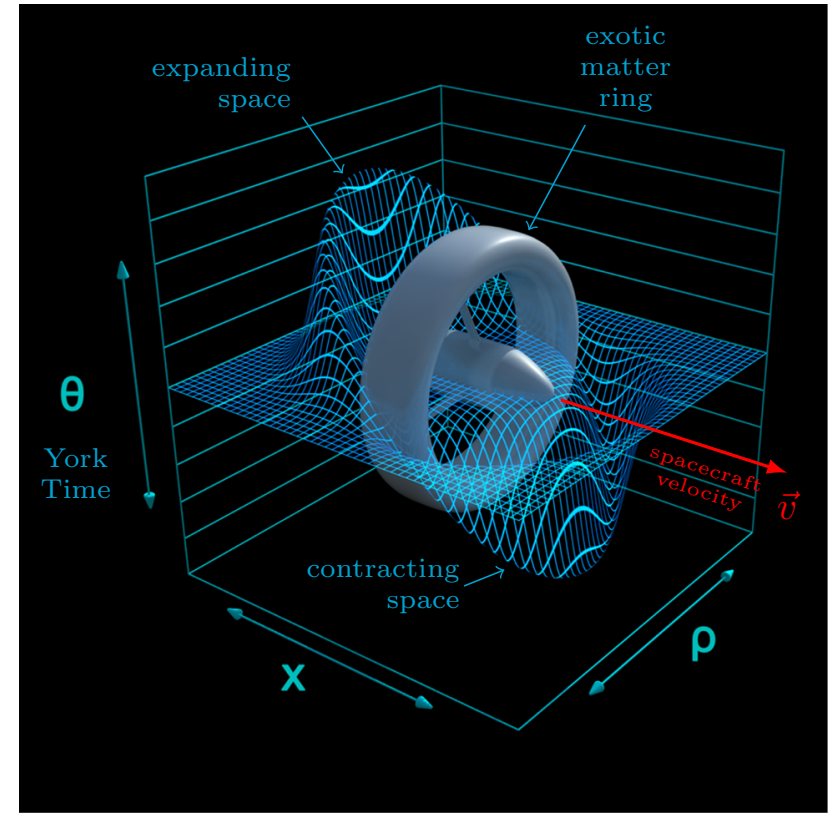

Fig. 8 Plot of York Time $\theta$ for Nature [17] - the plot shows the expansion and contraction of space associated with the Alcubierre metric. The plot also includes a simple representation of craft overlayed to illustrate the alignment of the exotic matter ring $\left(T^{00}\right)$ with the expansion and contraction of space. It should be noted that the flat region at the middle of the York Time plot represents the region of flat spacetime in the middle of the warp bubble where coordinate time is the same as proper time, and proper acceleration $\alpha$ is zero. This would be where sensitive spacecraft systems/instruments and possibly crew would be located. The effective velocity of the spacecraft is overlayed as a red vector

for the Casimir cavity is in effect a linear extrusion extending up from the surface of the paper meaning the lenticular shaped concentration is rod-like, while the Alcubierre plot is a revolution which yields a toroidal distribution.

Based on the custom Casimir cavity results for the parallel plate cavity with a cylindrical pillar at the mid-plane, a toy model comprised of a $1 \mu \mathrm{m}$ diameter sphere suspended in the middle of a $4 \mu \mathrm{m}$ diameter cylinder was implemented and the numeric worldline analysis technique was used find the predicted Casimir energy density. Figure 10a shows a section cut of the toroidal Casimir energy density for the spherecylinder system which correlates well with the toroidal Alcubierre energy density requirements. If one could manufacture a chip with these types of nano structures (nano-spheres suspended in nano-tubes), an experiment might be designed and attempted to conduct a test to measure transit time of say a current (alternately a photon or electron) through a tiny conductor (alternately open bore) routed through the center of the sphere(s). This transit time could be compared to the time it takes for a current (photon/electron) to run through a mirror system that has no external tube (control test). If need be, many of these nano structures could be arranged in parallel to increase the time resolution of the notional experiment 


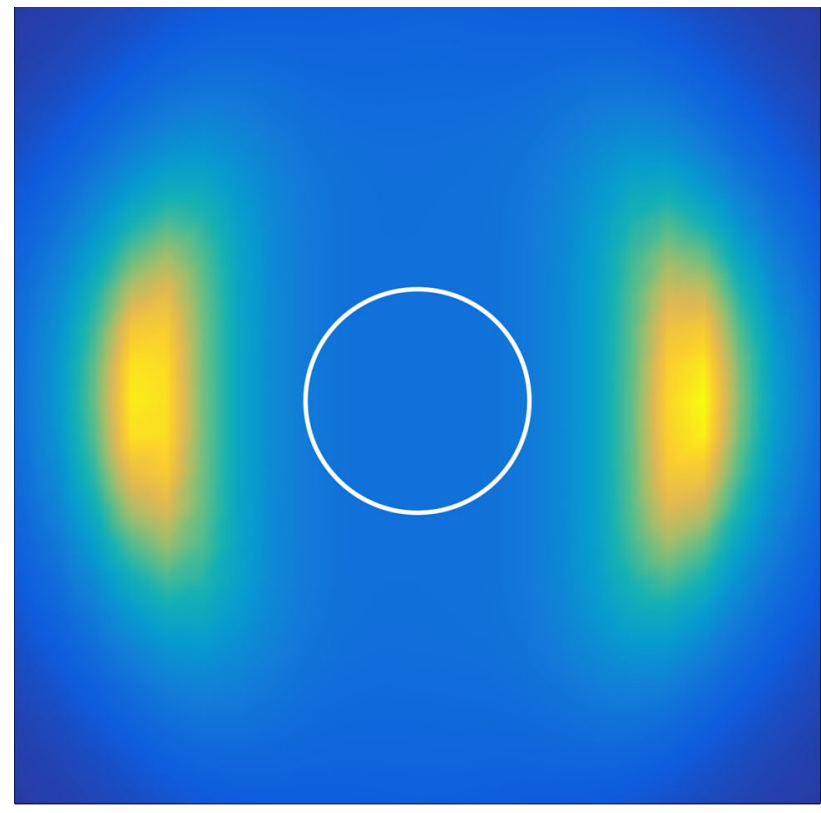

(a) close view of worldline numerics energy density result around pillar

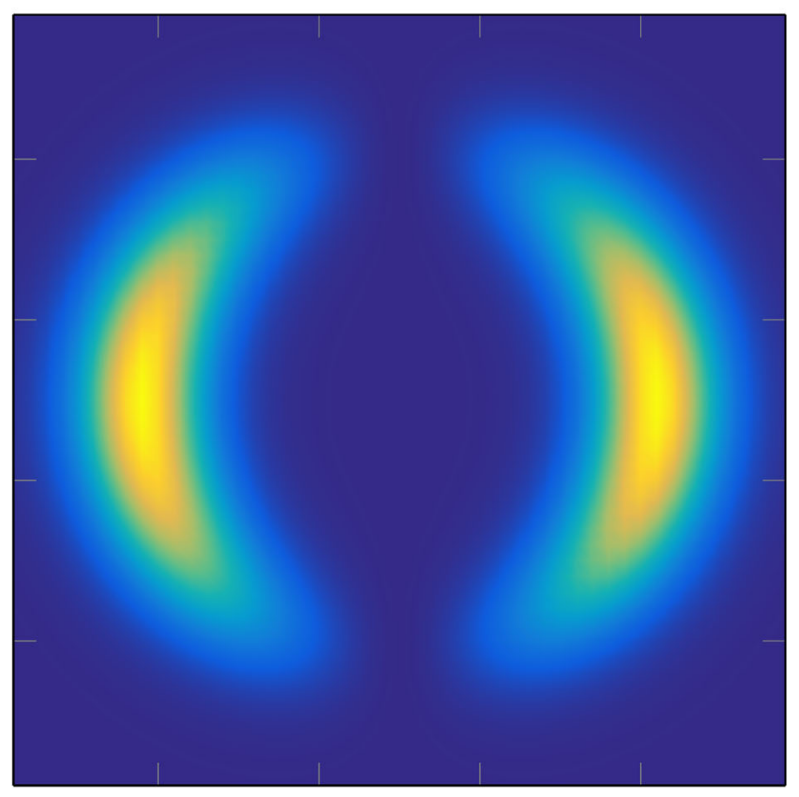

(b) $T^{00}$ energy density plot for Alcubierre metric

Fig. 9 Comparison of energy density for the numeric worldline assessment of the custom Casimir cavity and and the Alcubierre model energy density requirement

(see Fig. 10b). If a difference in transit time were observed, this would be an empirical confirmation of the generation of a real nano scale warp bubble on a chip. ${ }^{9}$ To be clear, this

\footnotetext{
${ }^{9}$ It could be speculated that a nano sphere might be made to translate through a nano cylinder as a more direct implementation of the Alcubierre model with the provision that it may be viewed as a space warp/wormhole hybrid with the cylinder serving as the connecting
}

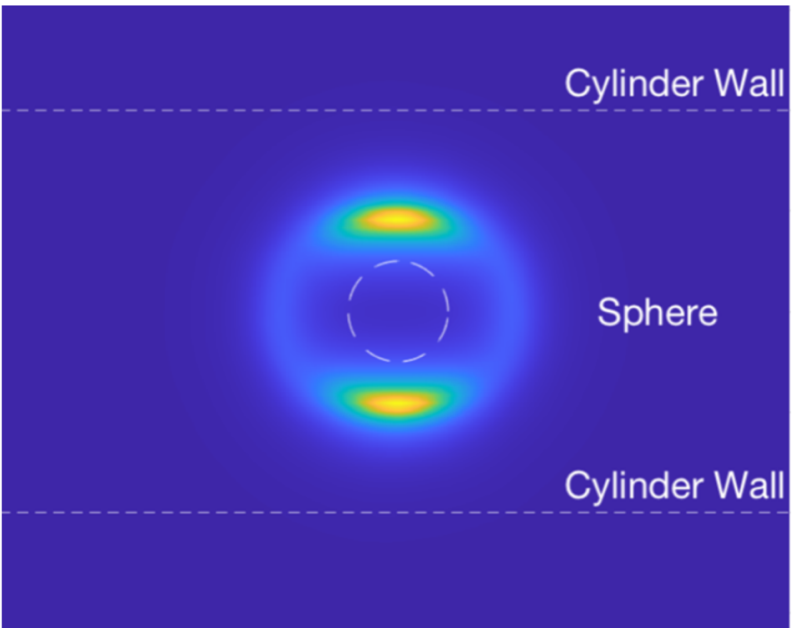

(a) Section cut of predicted toroidal Casimir energy density distribution for sphere-cylinder system

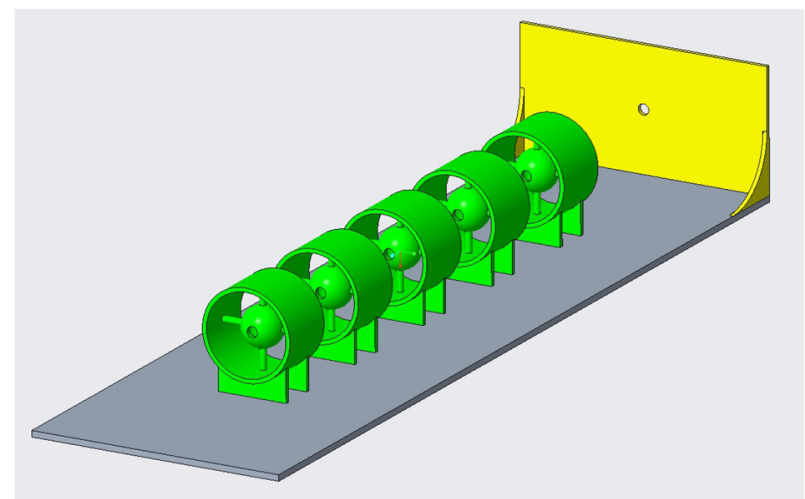

(b) Notional micro/nano structure depicting array of spherecylinder construction for possible experiment

Fig. 10 The top panel depicts a section cut of predicted toroidal Casimir energy density distribution for sphere-cylinder system comprised of a $1 \mu \mathrm{m}$ diameter sphere suspended in the middle of a $4 \mu \mathrm{m}$ diameter cylinder. The approach was implemented using Open MPIenabled c-code and the analysis was run on $6602.40 \mathrm{GHz}$ Intel Skylake CPUs. The model grid was a $100 \times 100$ grid with a 2000 unit-loop ensemble. The bottom panel depicts a notional experimental setup that comprises an array of sphere-cylinder constructions arranged such that a pulse of electrons or photons may be routed through the test device in an attempt to measure a change in transit time for an equivalent pulse routed through free space

would not be some simple analogue or proxy representation of a space warp phenomenon, rather it would be a genuine implementation of the idea in physical fact with observable consequences in the laboratory - just not in the dramatic form of a craft bound for a distant stellar destination.

\section{Footnote 9 continued}

pathway between two points and also enabling the formation of the necessary negative vacuum energy density around the sphere to boost the effective velocity. 


\section{Conclusions}

The impetus for the work discussed in this manuscript was to explore the implications of the dynamic vacuum model as applied to a custom Casimir cavity geometry. The dynamic vacuum model suggests that the state of the negative vacuum energy density in the cavity is not just an isotropic value that is constant throughout the enclosing geometry, rather it has spatial variation and can manifest complicated structure. In the process of the team exploring the literature, a technique called worldline numerics was discovered that also predicts that there is structure to the perturbed vacuum state that is predicted to exist in a notional Casimir cavity. This technique was used to evaluate the predicted state of the vacuum in response to the presence of small pillars placed at the midplane of a Casimir cavity. The analysis showed that the pillar would not adversely screen itself from the predicted background field that exists in response to just the presence of the plates. The analysis also showed a possible intersection with a model developed in the context of general relativity to understand how hyperfast stellar travel might be manifested mathematically. The qualitative correlation would suggest that a chip-scale experiment might be explored to attempt to measure a tiny signature illustrative of the presence of the conjectured phenomenon.

Acknowledgements This work was supported by the DARPA Defense Science Office Quest for Undiscovered Energy Storage and Thrust (QUEST) Program through funded agreement HR00112090082. Special thanks goes to Limitless Space Institute for organizational and institutional support, Prof. Mike Fiddy and Dr. David Lewis for helpful technical discussions, and Mark Rademaker for help with visualization graphics (e.g. Fig. 8). Godspeed.

Data Availability Statement This manuscript has no associated data or the data will not be deposited. [Authors' comment: This paper is primarily a theoretical work. The algorithms used have been summarized in the text and references to the salient literature have been provided. All necessary data related to the article are presented and discussed in the narrative.].

Open Access This article is licensed under a Creative Commons Attribution 4.0 International License, which permits use, sharing, adaptation, distribution and reproduction in any medium or format, as long as you give appropriate credit to the original author(s) and the source, provide a link to the Creative Commons licence, and indicate if changes were made. The images or other third party material in this article are included in the article's Creative Commons licence, unless indicated otherwise in a credit line to the material. If material is not included in the article's Creative Commons licence and your intended use is not permitted by statutory regulation or exceeds the permitted use, you will need to obtain permission directly from the copyright holder. To view a copy of this licence, visit http://creativecomm ons.org/licenses/by/4.0/.

Funded by SCOAP ${ }^{3}$.

\section{References}

1. H. White, J. Vera, P. Bailey, P. March, T. Lawrence, A. Sylvester, D. Brady, Dynamics of the vacuum and Casimir analogs to the hydrogen atom. J. Mod. Phys. 6, 1308-1320 (2015)

2. H. White, P. Bailey, J. Lawrence, J. George, J. Vera, A discussion on a dynamic vacuum model: derivation of Helmholtz equation from Schrödinger equation. Phys. Open 1, 100009 (2019)

3. G. Moddel, A. Weerakkody, D. Doroski, D. Bartusiak, Opticalcavity-induced current. Symmetry 13, 517 (2021)

4. H. White, P. Bailey, J. Lawrence, J. George, J. Vera, Dynamic vacuum model and Casimir cavity experiments. JBIS 73, 7 (2020)

5. H. Gies, K. Langfeld, L. Moyaerts, Casimir effect on the worldline. JHEP 2003, 6 (2003)

6. H. Gies, K. Klingmüller, Worldline algorithms for Casimir configuration. Phys. Rev. D 74, 4 (2006)

7. H. Gies, K. Klingmüller, Casimir edge effects. Phys. Rev. Lett. 97, 22 (2006)

8. K. Klingmüller, Worldline approach to Casimir effect and GrossNeveu model, Ph.D.-thesis. Heidelberg University (2007)

9. K. Aehlig, H. Dietert, T. Fischbacher, J. Gerhard, Casimir forces via worldline numerics: method improvements and potential engineering applications (2011). arXiv:1110.5936 [hep-th]

10. L. Moyaerts, K. Langfeld, H. Gies, Worldline approach to the Casimir effect. 6th Workshop on Quantum Field Theory under the Influence of External Conditions (QFEXT03), 11 (2003). arXiv:hep-th/0311168

11. M.S. Morris, K.S. Thorne, Wormholes in spacetime and their use for interstellar travel: A tool for teaching general relativity. Am. J. Phys. 56, 5 (1988)

12. M.S. Morris, K.S. Thorne, U. Yurtever, Wormholes, time machines, and the weak energy condition. Phys. Rev. Lett. 61, 13 (1988)

13. M. Visser, Lorentzian Wormholes: From Einstein to Hawking (American Institute of Physics, New York, 1995)

14. M. Alcubierre, The warp drive: hyper-fast travel within general relativity. Class. Quantum Gravity 11, 5 (1994)

15. R. Garattini, Casimir wormholes. Eur. Phys. J. C 79, 11 (2019)

16. R. Garattini, Generalized absurdly benign traversable wormholes powered by Casimir energy. Eur. Phys. J. C 80, 12 (2020)

17. S. Perkowitz, Science Fiction: Boldly Going for 50 Years (2016). https://www.nature.com/articles/537165a

18. H. White, A discussion of space-time metric engineering. Gen. Relativ. Gravit. J. 35, 2025 (2003)

19. H. White, Warp field mechanics 101. J. Br. Interplanet. Soc. 66, $242(2013)$

20. H. White, Warp Field Mechanics 102 (2013). https://ntrs.nasa.gov/ citations/20130011213

21. H. White, Shell thickness parameter $\sigma$ sensitivity analysis of York Time for Alcubierre model, animation of results (2013). https:// www.youtube.com/watch?v=gy_UFmGh83E

22. H. White, Shell thickness parameter $\sigma$ sensitivity analysis of $T^{00}$ for Alcubierre model, animation of results (2013). https://www. youtube.com/watch?v=n_VQPqKUwhQ 